\title{
Antwort zum Kommentar zum Artikel „Psychopharmaka und Diabetes“" von Ress C, Tschoner A, Kaser S und Ebenbichler CF. In Wiener Medizinische Wochenschrift 2011;161/21-22:531-542
}

\section{Christoph Ebenbichler}

Eingegangen: 17. Oktober 2012 / Angenommen: 18. Oktober 2012 / Online publiziert: 28. November 2012

(C) Springer-Verlag Wien 2012

Zuallererst möchten wir uns für den Kommentar bedanken und durchaus Ihrer Aussage zustimmen, dass das Thema „Psychopharmaka und Diabetes" ein sehr komplexe und klinisch bedeutsame Materie darstellt.

Die Hauptkritik dieses Kommentares bezieht sich auf die Tab. 4 des Artikels. Bei Tab. 1, ehemals Tab. 4, handelt es sich um eine sehr komplexe Tabelle.

Wie oben dargestellt, bringt diese Tabelle einen Überblick über Antidepressivagruppen mit deren einzelnen Vertretern, die dann nicht weiter unterschieden werden. Dementsprechend werden in dieser Übersichtstabelle generelle Aussagen zu Gruppen von Antidepressiva getätigt, was naturgemäß die Aussagen über die einzelnen Substanzen einschränkt.

Die MAOI Phenelzin und Tranylcypromin sollten natürlich differenziert werden, wobei die klinischen Studien zu diesen Substanzen wenig aussagekräftig und mit geringer Patientenanzahl sind, eine Problematik, die generelle Aussagen zu manchen Substanzen einfach nicht zulässt, aber zumindest gewisse Trends erkennen lässt.

Wegen des hohen Nebenwirkungspotentials sind sowohl Phenelzin als auch Tranylcypromin in der Schweiz und Österreich nicht mehr am Markt befindlich.

$\mathrm{Zu}$ bemerken ist sicherlich auch einen gewisser ,Interessenskonflikt', der diesem Kommentar anhaftet, da der Autor in der Wissenschaftlichen Abteilung der Firma Aristo Pharma $\mathrm{GmbH}$, dem Hersteller von Tranylcypromin, tätig ist.

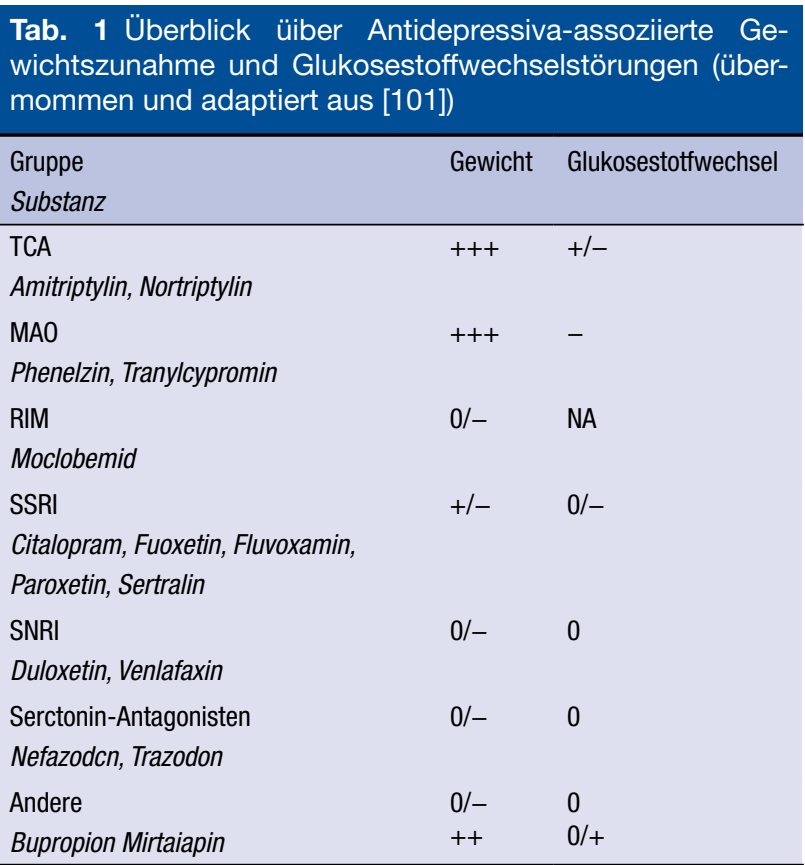

Gewicht +++ deutliche Gewichtszunahme, ++ geringe Gewichtszunahme, 0/+ minimale Gewichtszunahme, +/- uneinheitliche Gewichtszunahme/-abnahme, 0/- minimale Gewichtsabnahme. Glukosestoffwechse/ $0 /+$ nicht eindeutig belegter Anstieg der Plasmaglukose (PG), +/- uneinheitliche PG-Änderung, 0/- nicht eindeutig belegter Abfall der PG, - Abfall der PG, 0 keine Asscziation mit PG-Änderungen, NA nicht bekannt. MAOI Monoaminooxidaseinhibitoren, RIMA reversible Inhibitonen der Monoaminooxidase A, SNRI Serotonin- und Noradrenalin-Reuptake-Inhibitoren, SSRI selektive Serotonin-Reuptake-Inhibitonen, TCA trizyklische Antidepressiva

\footnotetext{
ao. Univ.-Prof. Dr. C. Ebenbichler $(\bowtie)$

Universitätsklinik für Innere Medizin I, Medizinische Universität Innsbruck, FA für Innere Medizin, Anichstraße 35, 6020 Innsbruck, Österreich

E-Mail: christoph.ebenbichler@i-med.ac.at
} 